\title{
Health Issues in Rohingya Refugees in Bangladesh:
}

\section{Ahmed N ${ }^{1}$, Rahman MM²}

Refugees are those unfortunate people, who migrated from their motherland to some other country due to some kind of unfavourable conditions. Rohingyas are an ethnic, linguistic and religious minority group of Northern Rakhine State (NRS) of Myanmar. Myanmar governments categorized them as illegal immigrants from Bangladesh and have been excluded from citizenship and basic human rights. From 1991-1992 a mass exodus of more than 250,000 Rohingya regugees fled persecution in the union of Myanmar and arrived in Bangladesh, living in temporary camps. Since late August 2017 more than 613,000 Rohingya muslims have fled Myanmar's Rakhine state to escape the military's large scale campaign of ethnic cleansing. The atrocities committed by Myanmar security forces, including mass killing, sexual violence, and widespread arson, amount to crime s against humanity. Rohingya refugees are one of the most vulnerable groups due to lack of health care system, personal hygiene, shelter, sanitation and violence. It is evident that these refugees suffer from a variety of health problems because of their living conditions \& environmental sanitation is not similar to an independent nation. Further, basic amenities like medicines \& other services are not available.

Around 1.2 million people are estimated to be in need of health assistance. This number includes both newly arrived Rohingyas from Myanmar since 25 August, and their host communities. Based on the public health situation analysis published on 10 October 2017, WHO has graded this crisis as a level 3 emergency, the highest possible rating. Rohingyas are a heavy burden to our government health care facilities. Sadar Hospital in Cox's Bazar and Teknaf and Ukhia upazilla health complexes and primary health care (PHC) units are not equipped to handle the huge influx of Rohingya refugees, and are running substantially over capacity to meet the needs of both the new arrivals and the host populations in the area. The Rate of severe acute malnutrition (SAM) are running at 7.5\%, well over the emergency threshold. Local health care facilities and NGOs have limited capacity to treat children with SAM with complications. Although the Government of Bangladesh has allocated 2000 acres for a settlement area for the new arrivals, the earmarked land consists of remote, hilly terrain that has no established infrastructures and no
[J Shaheed Suhrawardy Med Coll 2017; 9(1): 1-2] DOI: http://dx.doi.org/10.3329/jssmc.v9i1.37249

roads linking it to Cox's Bazar or other towns creates inequitable access to services. Health care services are currently concentrated in the more accessible areas of the camps. As a result, some areas are over-served while in other areas have no or very limited access to health care. Crowded living conditions, inadequate water and sanitation (WASH) facilities and low vaccination coverage present significant risks of communicable disease outbreaks. As of 11 November, 611 suspected cases of measles were reported. The Cox's Bazar area is prone to cyclones that can potentially destroy temporary shelters and displace large segments of population. The incidence of acute respiratory infections (ARIs) is expected to rise with the onset of winter. Due to complete deforestation and topography of the camps, there is a risk of landslides in the next rainy seasons. Essential reproductive health/ maternal child and newborn health services, particularly obstetric services, are inadequate either due to insufficient bed space or lack of facilities in hard-to-reach areas. Home deliveries are anecdotally reported to be high. The needs of mental and psychosocial health care service for Rohingyas are immense. Many Rohingya are reported to have been physically and mentally traumatized by the violence, including sexual and gender-based violence (SGBV).

A multi-sectoral humanitarian response plan covering the period September 2017 to February 2018 was published in early October 2017. The plan focuses on three strategic objectives: 1) provide life-saving basic assistance in settlements, camps and host communities; 2) improve conditions in and management of both existing and new settlements, including infrastructure and site planning; and 3) promote safety, dignity and respect for the individual rights of the affected population.

There are two health sector sub-groups: one on sexual and reproductive health (SRH) led by UNFPA, and one on mental health and psychosocial support (MHPSS) led by ACF. A multi-sectoral acute watery diarrhea preparedness and response plan working group (AWD-PRP WG) was established and meets regularly. The health sector actively collaborates with the Nutrition, WASH and Logistic sectors. 
The health sector has designed a recommended package of minimum basic health services for health posts and health care centres, based on the MOHFW's service delivery package and UNHCR/SPHERE guidelines. The package has been approved by MOHFW and shared with all health sector partners to inform their programming.

Additionally, since 1 November, 970 children (6 months 15 years) passing through the two transit sites have been vaccinated against measles and rubella (MR) and 1038 children under five years received oral polio vaccine (BOPV)

Needless to say that there is a high risk of spreading of contagious diseases like diphtheria, measles, rubella and HIV from the Rohingya people to Bangladeshis. Also massive deforestation are being carried out, which is very harmful for the environment. Our main tourist spot Cox's Bazar is under a great threat due to huge influx of Rohingya in that area. So intrusion of more and more Rohingya refugees is a threat for our social, economic, environmental and health equilibrium.

Rohingya refugees are far away from achieving their basic human rights. They have been stateless without a legal nationality from 1962. We advocate supply of adequate medicine, increase health care providers, increase hospital services, decreased pollution, adequate supply of safe drinking water, improved sanitation and increase awareness to physical and mental health.

[J Shaheed Suhrawardy Med Coll 2017; 9(1): 1-2]

1. Dr. Nadim Ahmed, Senior Consultant (Surgery), Shaheed Suhrawardy Medical College \& Hospital

2. Prof. Mustafizur Rahman, Professor (Surgery), Shaheed Suhrawardy Medical College \& Hospital 